\title{
Diseño e implementación de unidades de conversión de energía eléctrica para uso en redes de generación a partir de fuentes no convencionales
}

\author{
Design and implementation of units of conversion \\ of electrical energy for using in generation networks, \\ from non-conventional sources
}

Fecha de recepción: 30 de marzo de 2007

Fecha de aceptación: 13 de julio de 2007
Cesảr Leonardo Trujillo Rodríguez
Rafael Antonio Peña Suesca ${ }^{2}$
Javier Antonio Guacaneme Moreno ${ }^{3}$

$\int_{\text {tran }}^{\text {R }}$

\section{RESUMEN}

En muchos lugares del mundo existen zonas adonde es realmente complejo transportar la energía eléctrica. Para estos casos, una solución es proveerse de sistemas propios de generación, entre los cuales puede considerarse el aprovechamiento de fuentes de energía, posiblemente no convencionales, presentes en estas zonas. Una alternativa es el diseño de un sistema que transforme y almacene energía en un banco de baterías y, a partir de este, convertirla en valores estándar A, C (por ejemplo, $120 \mathrm{~V} / 60 \mathrm{~Hz}$ ). Dichos procesos de

1 Ingeniero electrónico Universidad Distrital Francisco José de Caldas. Magister en Ingenieria Eléctrica Universidad Nacional de Colombia. Profesor Universidad Distrital Francisco José de Caldas. Universidad Distrital Francisco José de Caldas - Línea de Investigación en Fuentes Alternas de Energía LIFAE

cltrujillo@udistrital.edu.co

2 Ingeniero electrónico Universidad Distrital Francisco José de Caldas. Magíster en Ingeniería Eléctrica Universidad Nacional de Colombia. Profesor Universidad Distrital Frincisco José de Caldas.

rpena@udistrital.edu.co

3 Ingeniero electrónico Universidad Distrital Francisco José de Caldas. Magíster en Ingeniería Eléctrica Universidad Nacional de Colombia. Profesor Universidad Distrital Francisco José de Caldas. jguacaneme@udistrital.edu.co 
conversión deben ser muy eficientes, porque en general se trata de energía disponible durante ciertos periodos, en muchos casos escasa y costosa.

En el presente artículo se analiza la etapa de conversión a partir de un bus DC el cual provee un medio de almacenamiento de la energía que se recolecta a través de algún tipo de fuente. Así mismo se analiza lo relacionado con los principios de operación, dimensionamiento de los componentes y características de diseño para la implementación de unidades electrónicas de conversión DC/AC de baja potencia, capaces de manejar potencia activa y reactiva en ambas direcciones (desde las baterías hacia la red y viceversa) y con posibilidad de interconexión a otros sistemas con generación. Además se presenta un análisis práctico del desempeño de la unidad electrónica.

\section{Palabras clave}

Conversión de potencia bidireccional, fuentes no convencionales, modulación de ancho de pulso sinusoidal.

\section{ABSTRACT \\ In many places of the world there are zones where it is really complex to transport the electrical energy. In these cases, a solution is to provide itself with own systems of generation in which the advantage of non-conventional power sources presented in these zones can be considered. An alternative is the design of a system that transforms and stores energy in a bank of batter- ies and, from this one, to convert it to values standard AC (e.g. $120 \mathrm{~V} / 60 \mathrm{~Hz})$. These conversions must be highly efficient, since generally it is energy avail- able during certain periods, in many cases little and expensive one.}

In this paper is analyzed the convertion stage from a $\mathrm{DC}$ bus which provides a storage media of the energy that is collected through some type of source. They are also analyzed the operation principles, sizing of the components and characteristics of design for the implementation of DC/AC electronic units of conversion of low power able to handle active power and reactive power in both directions (from the batteries towards the network and vice versa) and with possibility of interconnection to others generation systems. Moreover, a practical analysis of the performance of the electronic unit is described. 


\section{Key words}

Bidirectional power conversion, non-conventional sources, sinusoidal pulse width modulation.

\section{INTRODUCCIÓN}

Debido al incremento en la demanda de energía eléctrica, a que las fuentes tradicionales de generación no son suficientes y que existen regiones desatendidas por este servicio, ha sido necesario investigar en nuevas fuentes de energía, como el viento y el sol.

Debido a que la energía eléctrica presenta ventajas como fácil transporte, distribución y conversión en otros tipos de energía, la energía colectada de las fuentes no convencionales es transformada en energía eléctrica DC que presenta ventajas para la interconexión y, en algunos casos, es almacenada en baterías para uso posterior. Es necesario disponer de sistemas que conviertan esta energía $\mathrm{DC}$ en energía $\mathrm{AC}$; que para el caso de bajas potencias, se requiere a 208/120 $\mathrm{V}_{\mathrm{rms}} / 60 \mathrm{HZ}$. Aparte de realizar dicha transformación estos sistemas, deben ser altamente eficientes y capaces de transferir energía hacia la red de energía alterna, dentro del principio de cogeneración.

Un sistema de generación y distribución de energía a partir de fuentes no convencionales tiene la característica de utilizar fuentes que no están disponibles las 24 horas del día, como en los casos del viento y del sol: Lo anterior es ventajoso cuando el sistema está interconéctado con una red de generación convencional que suple los requerimientos de energía en los momentos que no se tiene generación de las fuentes no convencionales. Los sistemas de generación no convencional aislados, que deban garantizar servicio continuo, requieren sistemas adicionales de generación o sistemas de almacenamiento de energía; por ejemplo, bancos de baterías.

Teniendo en cuenta lo anterior y las ventajas de las interconexiones DC, estos sistemas requieren conversores AC/DC y DC/AC. A continuación se analizará una unidad de conversión prototipo pequeña que permite la transferencia de energía de las fuentes $\mathrm{DC}$ a la red $\mathrm{AC}$, y viceversa. 


\section{UNIDAD DE CONVERSIÓN}

\section{Conversión DC/AC}

Se implementó mediante configuración inversora de puente completo [1], como se muestra en la figura 1.

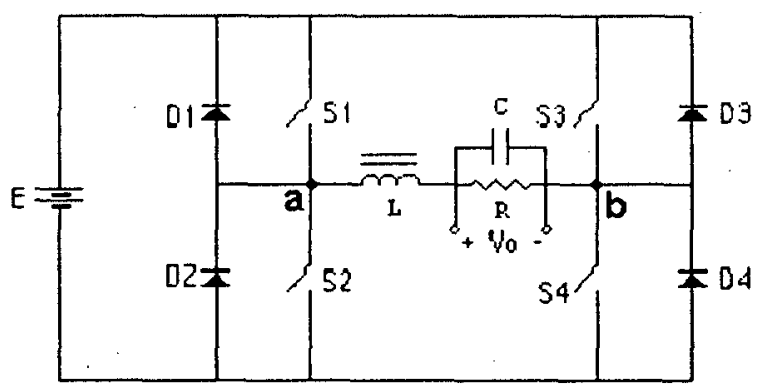

Figura 2 Forma de onda de $V_{a} b$.

La configuración anterior permite obtener sobre la carga voltajes $\mathrm{Vo}$, positivos, negativos o cero, según lo determine el circuito de control de los interruptores.

Funcionamiento bipolar

Si en el circuito de la figura 1 , en el tiempo $T 1$ están cerrados los interruptores $S 1$ y $S 4$, y abiertos $S 2$ y $S 3$, entonces $V_{a b}=E$. Si posteriormente en el tiempo $T 2$ se cierran los interruptores $S 2$ y $S 3$, y se abren $S 1$ y $S 4$, entonces $V_{a b}=-E$. La forma de onda de $V_{a b}$ se muestra en la figura 2.

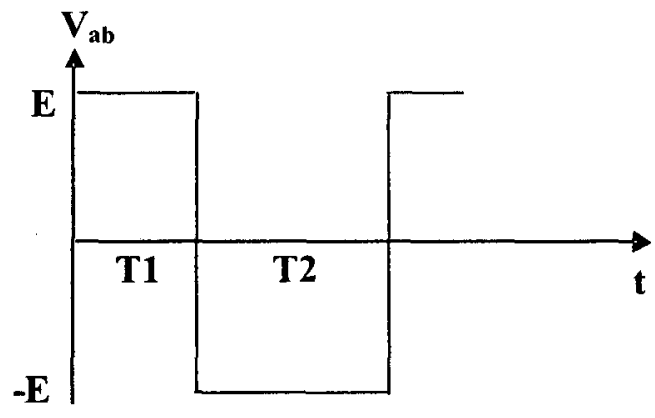

Figura 1 Puente completo. 
Para señales periódicas con $T=T 1+T 2$, el valor medio de $V_{a b}$ es

$$
V_{a b D C}=\frac{E \cdot T 1-E \cdot T 2}{T}
$$

Definiendo $T 1 / T$ como el ciclo útil ( $\delta$ ) de la señal, $V_{a b D C}$ se puede expresar como

$$
V_{a b D C}=E(2 \cdot \delta-1)
$$

Teniendo en cuenta que $\mathrm{L}$ es cortocircuito para DC, entonces

$$
V_{o D C}=V_{a b D C}
$$

es decir,

$$
V_{o}=E(2 \cdot \delta-1)
$$

Si el filtro pasabajos LC elimina las componentes AC, entonces el voltaje $V_{\text {。 }}$ es solo la componente DC. En la tabla 1 se muestran los valores de $V_{o}$ para diferentes valores de $\delta$.

Tabla 1.

$V_{0}$ en función de $\delta$

\begin{tabular}{|c|c|}
\hline Ciclo útil (8) & $\mathrm{V}_{\mathrm{o}}$ \\
\hline 0 & $-\mathrm{E}$ \\
\hline 0.25 & $-\mathrm{E} / 2$ \\
\hline 0.5 & 0 \\
\hline 0.75 & $\mathrm{E} / 2$ \\
\hline 1 & $\mathrm{E}$ \\
\hline
\end{tabular}

\section{Modulación SPWM}

En la práctica, la conmutación de los interruptores se realiza a alta frecuencia (de $1 \mathrm{kHz}$ a $20 \mathrm{kHz}$ ) y el filtro LC pasabajos se escoge para dejar pasar bajas frecuencias $(50 \mathrm{~Hz}$ o $60 \mathrm{~Hz}$ ) y bloquear las altas frecuencias. En estos casos el voltaje $V_{o}$ puede variar a baja frecuencia y la ecuación (4) se puede escribir

$$
V_{o}(\omega t)=E[2 \cdot \delta(\omega t)-1]
$$


Para obtener un voltaje sinusoidal de la forma

$$
V_{o}(\omega t)=E \sin (\omega t)
$$

se requiere

$$
\delta(w t)=0.5+0.5^{*} \operatorname{sen}(w t)
$$

que se obtiene despejando $\delta(\omega t)$ de las ecuaciones (5) y (6).

Como se observa en la ecuación (7), el ciclo útil (ancho de pulso) varía de acuerdo con una función sinusoidal. Esta técnica se denomina SPWM (Sinusoidal Pulse Width Modulation) [2]. Es decir se modula el ancho del pulso de una portadora de alta frecuencia $(20 \mathrm{kHz})$ con una señal sinusoidal moduladora de baja frecuencia $(60 \mathrm{~Hz})$.

Para la implementación de un sistema SPWM se debe tener en cuenta:

- Las frecuencias de la portadora y de la moduladora.

- Número de pulsos por ciclo de moduladora.

- Índice de modulación $\mathrm{m}=2 * \delta_{\mathrm{m}}, 0<\mathrm{m}<1$.

- Sistema de filtrado.

- Dispositivos utilizados para la implementación.

En el prototipo que se implementó, la generación de la señal SPWM se realizó digitalmente usando un microcontrolador. El proceso consistió en dividir el periodo de la señal sinusoidal en $p$ intervalos. Se discretizó la señal sinusoidal en 340 particiones para poder describirla como una sucesión de anchos de pulso variables en el tiempo.

\section{Descripción del prototipo}

La unidad de conversión prototipo es monofásica, de baja potencia $(400 \mathrm{~W})$ y presenta las siguientes características: 
- Conversión $48 \mathrm{~V}_{\mathrm{DC}} / 120 \mathrm{Vrms}$ sinusoidal regulada a $60 \mathrm{~Hz}$, y viceversa.

- Control de corriente AC constante cuando se presenta sobrecarga.

- Posibilidad de interconectarse con redes AC convencionales y con otros módulos semejantes.

Dimensionamiento de los dispositivos

Para la etapa de potencia de la figura 1, que funciona con modulación SPWM, los voltajes y corrientes en los dispositivos $\mathrm{S} 1, \mathrm{~S} 2, \mathrm{~S} 3$ y S4, idealmente, tienen las formas de onda que se muestran normalizadas en la figura $3 a$ y 3 b.
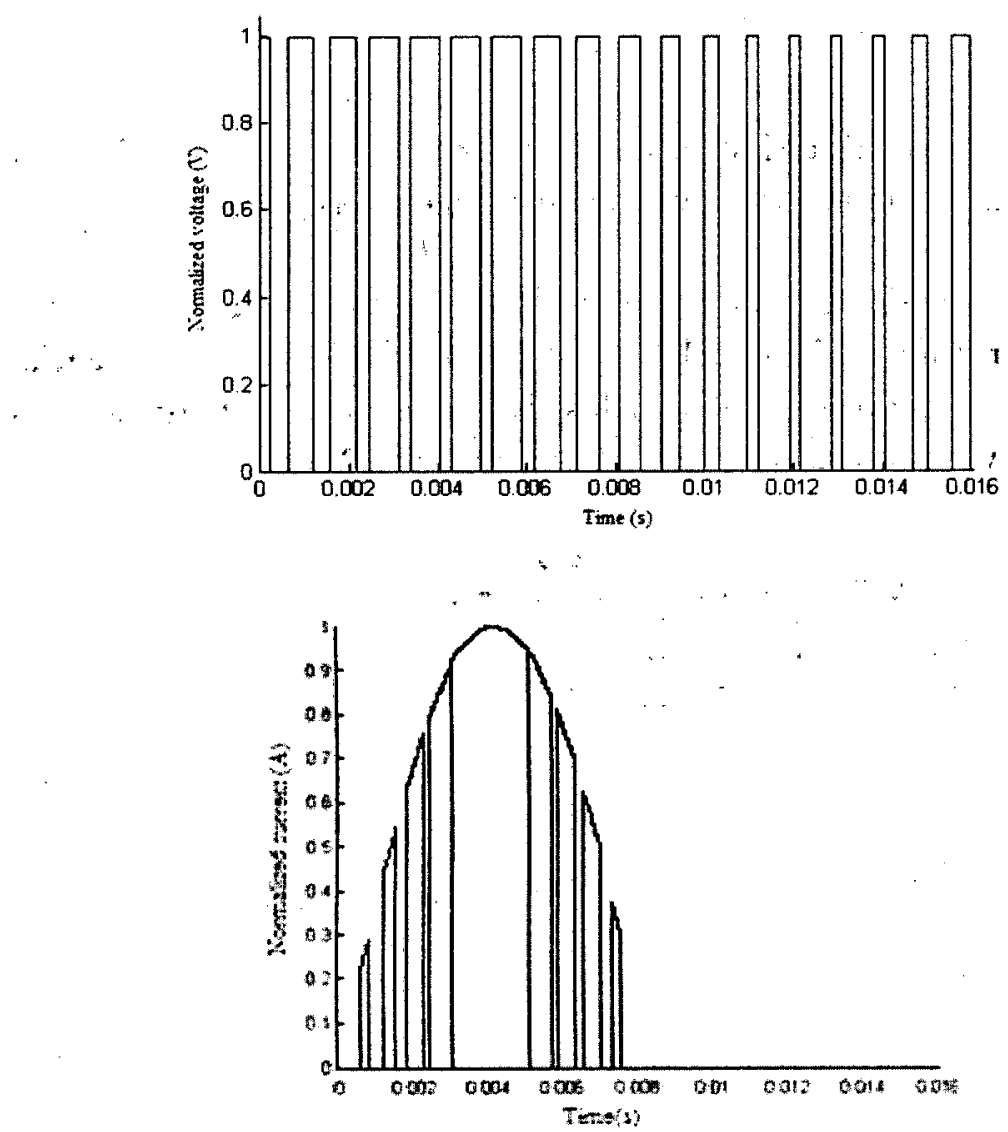

Figura 3 Formas de onda de a) voltaje y b) corriente sobre S1, S2, S3 y S4. 
En las ecuaciones (8) se presentan las expresiones de voltaje y corriente sobre los dispositivos S1, S2, S3 y S4 [3].

$$
\begin{aligned}
& V_{r m s}=E\left[\frac{1}{340} \sum_{n=1}^{340}\left(0.5+\frac{m}{2} \operatorname{sen}\left(\frac{n \cdot \pi}{170}\right)\right)\right]^{1 / 2}=\frac{E}{\sqrt{2}} \\
& V_{D C}=\frac{E}{340} \sum_{n=1}^{340}\left(0.5+\frac{m}{2} \operatorname{sen}\left(\frac{n \cdot \pi}{170}\right)\right)=\frac{E}{2} \\
& I_{r m s}=\left[\frac{I_{p}^{2}}{2 \pi} \sum_{n=0}^{169} \int_{\frac{n \pi}{170}}^{\frac{\pi}{170}\left(n+\delta_{n}\right)} \operatorname{sen}^{2}(\omega t) d \omega t\right]^{1 / 2}=0.4791569 I_{p} \\
& I_{D C}=\frac{I_{p}}{2 \pi} \sum_{n=0}^{169} \int_{\frac{\pi \pi}{170}}^{\frac{\pi}{170}\left(n+\delta_{n}\right)} \operatorname{sen}(\omega t) d \omega t=0.2822771 I_{p}
\end{aligned}
$$

$E$ representa el voltaje de las baterías, $I_{p}$ la corriente pico sobre el dispositivo y 340 es el número de particiones tomadas en un periodo.

La unidad prototipo funciona a partir de un banco de baterías de $48 V_{D C} y$ utiliza un transformador de relación 1 a 6.5 que suministra una potencia de $400 \mathrm{~W}$ a una tensión de $120 \mathrm{~V}_{\mathrm{rms}}$. En los dispositivos se obtuvieron los valores de voltajes y corrientes que se muestran en la tabla 2.

Tabla 2.

Valores de voltaje y corriente en los dispositivos.

\begin{tabular}{|c|c|c|c|c|c|}
\hline $\mathbf{V}_{\text {PICO }}$ & $\mathbf{V}_{\text {RMS }}$ & $\mathbf{V}_{\mathrm{DC}}$ & $\mathbf{I}_{\text {PICO }}$ & $\mathbf{I}_{\text {RMS }}$ & $\mathbf{I}_{\mathrm{DC}}$ \\
\hline $48 \mathrm{~V}$ & $34 \mathrm{~V}$ & $24 \mathrm{~V}$ & $33 \mathrm{~A}$ & $16 \mathrm{~A}$ & $9.4 \mathrm{~A}$ \\
\hline
\end{tabular}

Para la topología utilizada, con bajas tensiones y mayores corrientes, las pérdidas en los dispositivos aumentan y la eficiencia se reduce aproximadamente al $70 \%[4]$.

Los dispositivos se conmutan a frecuencias altas haciendo más evidente el efecto de las capacitancias e inductancias, originando sobretensiones. Por tanto, y para efectos de diseño, es conveniente escoger dispositivos que soporten picos varias veces superiores a la tensión de las baterías. 
Filtro de salida

La señal SPWM de frecuencia portadora $\left(f_{\mathcal{C}}\right)$ de $20 \mathrm{kHz}$ y frecuencia moduladora $(\mathrm{fm})$ de $60 \mathrm{~Hz}$ tiene el espectro que se muestra èn la figura 4 . La energía se concentra principalmente en $60 \mathrm{~Hz}$, y sus armónicos en $20 \mathrm{kHz}$, $40 \mathrm{kHz}$ y $60 \mathrm{kHz}$. De acuerdo con lo anterior, se escogió para el diseño del filtro una frecuencia de corte de aproximadamente $180 \mathrm{~Hz}$. Una simulación de la característica se observa en la figura 5.

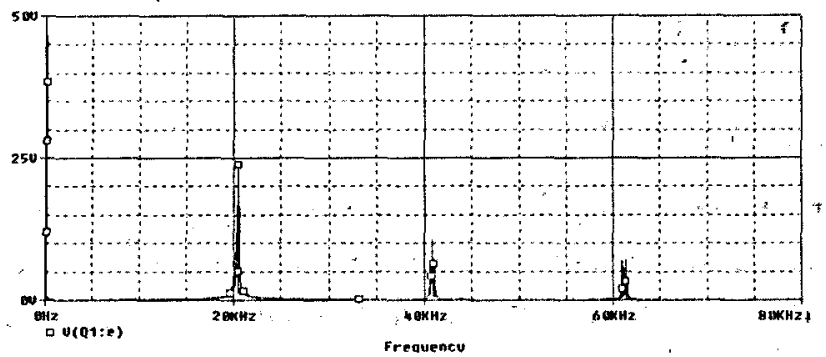

Figura 4 Espectro de la señal SPWM en frecuencia.

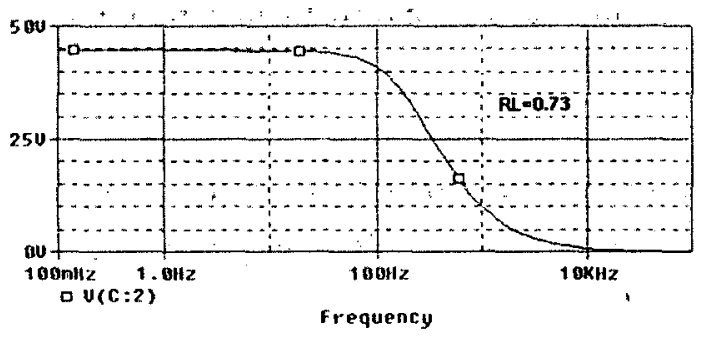

Figura 5 Respuesta en frecuencia del filtro a plena carga.

La topología del filtro es $\mathrm{LC}$, al cual se le han incluido la resistencia de pérdidas de la bobina $\left(R_{B}\right)$ y la resistencia de carga $R_{L}$ como se muestra en la figura 6.

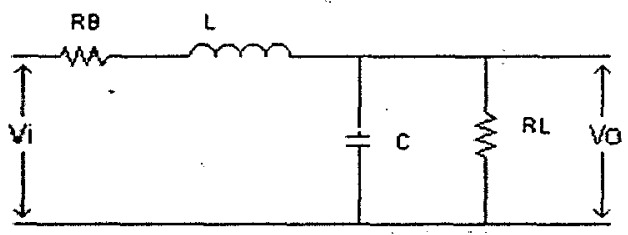

Figura 6 Filtro de salida. 
El cálculo se hizo estableciendo su función de transferencia, igualándola a $1 / \sqrt{ } 2$ (valor de media potencia) y calculando para $s=j \omega$. La función de transferencia se presenta en la ecuación (9):

$$
F(s)=\frac{V o(s)}{V i(s)}=\frac{R_{l}}{R_{L} L C s+s\left(R_{L} R_{B} C+L\right)+\left(R_{L}+R_{B}\right)}
$$

y se obtuvo el valor de $L=601 \mu \mathrm{H}$ para $R_{B}=0.06 \Omega, \mathrm{C}=25 \mu \mathrm{F}, f=200 \mathrm{~Hz}$ y $R_{L}=0.73 \Omega$ para $V_{o}=24 \mathrm{~V}_{\mathrm{rms}}$.

\section{Transformador de salida}

Sin considerar las pérdidas, idealmente el voltaje que puede generar el puente alimentado con el voltaje nominal de las baterías es $48 / \sqrt{2} \mathrm{~V}_{\mathrm{rms}}$. Considerando las pérdidas de voltaje en las baterías, el conversor $\mathrm{DC} / \mathrm{AC}$ y el filtro, el voltaje a la salida de la unidad es $24 V_{\text {rms }}$. Teniendo en cuenta que se requiere un transformador para elevar el voltaje a $120 V_{\text {rms }}$ y que para efectos de control es conveniente trabajar con índices de modulación inferiores a uno. Se escogió un transformador con relación de transformación de 6.5.

\section{Protecciones}

Debido a los sobrepicos de tensión por conmutación que se presentan en los dispositivos semiconductores, se requieren snubbers que limiten los voltajes máximo $\left(V_{\mathrm{pk}}\right)[5]$ (figura 7 ).

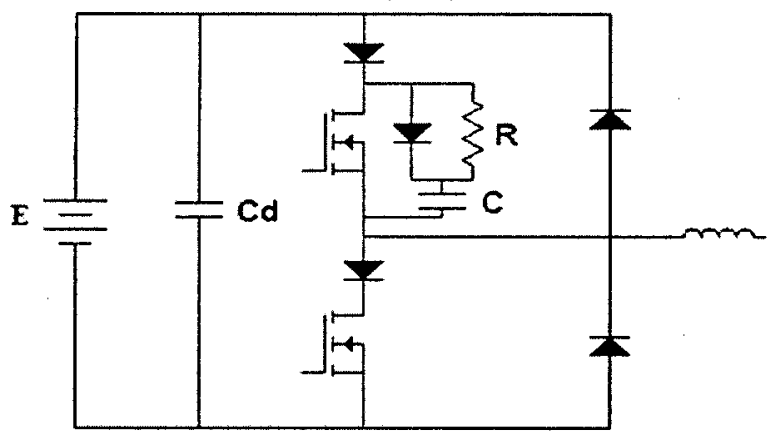

Figura 7 Snubbers. 
- Snubber Cd: corresponde al condensador colocado lo más cerca posible y en paralelo a la rama de los interruptores. Se calcula mediante la ecuación (10):

$$
C d=\left(L_{S}+L_{C}+L_{B}\right) I_{O}^{2} /\left(V_{p k}-V c c\right)^{2} \quad ;
$$

donde $L_{S}$ : es la inductancia del dispositivo, $L_{C}$ : es la inductancia de los conductores y $L_{B}$ es la inductancia de la bobina.

- Snubber de carga y descarga: se compone de un circuito RC y un diodo. El condensador y la resistencia se pueden calcular a partir de la ecuación (11):

$$
\frac{1}{2} L_{T} I_{O}^{2}=\frac{1}{2} C\left(V_{p k}-V c c\right)^{2} \quad y \quad R=\frac{1}{\left(6 \cdot C \cdot f_{S}\right)}
$$

donde $L_{T}$ es la inductancia parásita del circuito cuando el interruptor del

- lado inferior se encuentra encendido; $y f_{s}$ es la frecuencia de conmutación.

\section{Conversión AC/DC}

Para explicar el comportamiento de la unidad, es conveniente modelar el sistema como se muestra en la figura 8, donde $V_{\mathrm{TH}}$ y $Z_{\mathrm{TH}}$ es el equivalente Thevenin de la unidad de conversión, $\mathrm{V}$ y $\mathrm{Z}$ es el equivalente de la fuente externa. Para facilidad del análisis, se asume que tanto $\mathrm{V}_{\mathrm{TH}}$ como $\mathrm{V}$ son voltajes sinusoidales de $60 \mathrm{~Hz}$.

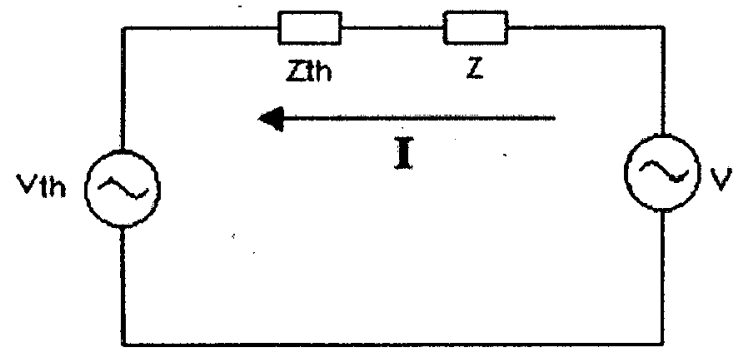

Figura 8 Modelo de la unidad electrónica conectada a un sistema externo. 
El flujo de potencia activa y potencia reactiva entre $\mathrm{V} \mathrm{y}_{\mathrm{TH}}$ depende de los valores de voltaje y fase de las fuentes y de los valores de la impedancia $\mathrm{Z}_{\mathrm{TH}}+$ $Z$. Para el caso de impedancia puramente reactiva, las potencias están dadas por la expresión (12), donde la transferencia de potencia activa depende principalmente de las diferencias angulares, La transferencia de potencia reactiva depende de la diferencia de las magnitudes de voltaje [6].

$$
P=\frac{V_{A} V_{B}}{X} \operatorname{sen}\left(\varphi_{A}-\varphi_{B}\right) \quad y \quad Q=\frac{V_{A}}{X}\left(V_{A}-V_{B} \cos \left(\varphi_{A}-\varphi_{B}\right)\right)
$$

En el caso de impedancias puramente resistivas, la potencia activa depende principalmente de las diferencias de voltaje; la potencia reactiva, de las diferencias angulares. En los casos intermedios, en donde la parte resistiva y reactiva es igualmente importante, las potencias activas y reactivas dependen de las diferencias de voltaje y de las diferencias angulares.

Para el prototipo implementado se realizaron ensayos de transferencia de potencia desde la red a las baterías, variando en primer lugar la magnitud de $\mathrm{V}_{\mathrm{TH}}$, en fase con $V$, y variando en segundo lugar la fase $\mathrm{de}_{\mathrm{TH}}$, con magnitud constante.

Las variaciones de magnitud y fase $\mathrm{de}_{\mathrm{TH}}$ se realizaron modificando el índice de modulación $m$ y el desfasamiento $\varphi$ de la expresión (13) sobre la que se puede realizar el control.

$$
\begin{aligned}
& V_{t h}=m \cdot a \cdot E \cdot \operatorname{sen}(\omega t+\varphi) \\
& a: \text { relación del transformador }
\end{aligned}
$$

En las figura 9 y 10 se muestran los resultados de los ensayos realizados sobre la unidad.

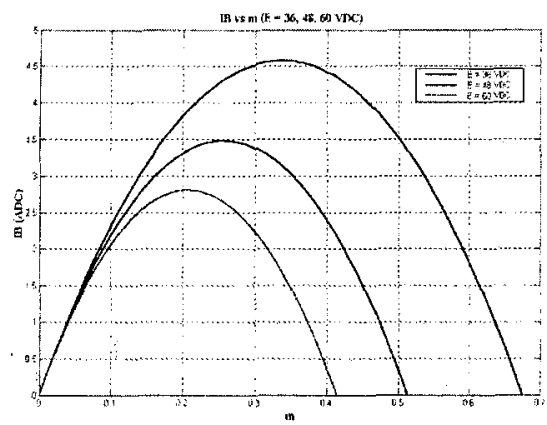




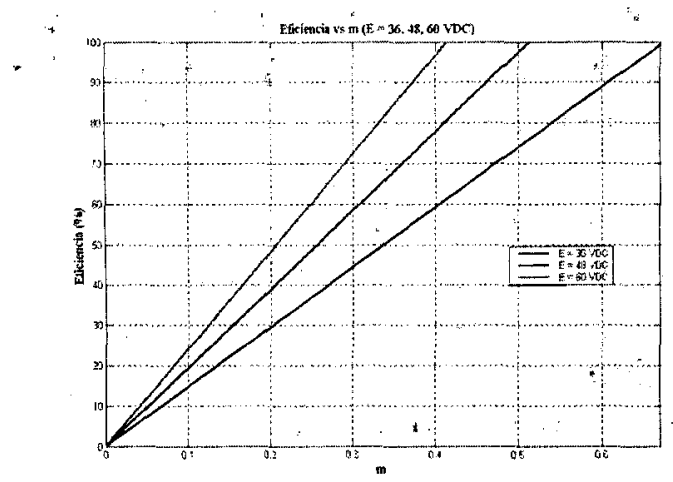

Figura 9 Con desfasamiento $\varphi=00$.a) Corriente en la batería $I_{B}$ vs. $m$ para $E=36 V_{D C^{\prime}}$ $48 \mathrm{~V}_{\mathrm{DC}}$ y $60 \mathrm{~V}_{\mathrm{DC}}$. b) Eficiencia vs. $\mathrm{m}$ para $\mathrm{E}=36 \mathrm{~V}_{\mathrm{DC}} 48 \mathrm{~V}_{\mathrm{DC}}$ y $60 \mathrm{~V}_{\mathrm{DC}}$

En la figura 9 se observa qùe en las corrientes máximas para las cuales corresponde una potencia máxima de $168 \mathrm{~W}$, aproximadamente; el equipo presenta una eficiencia del $50 \%$. $\therefore$,
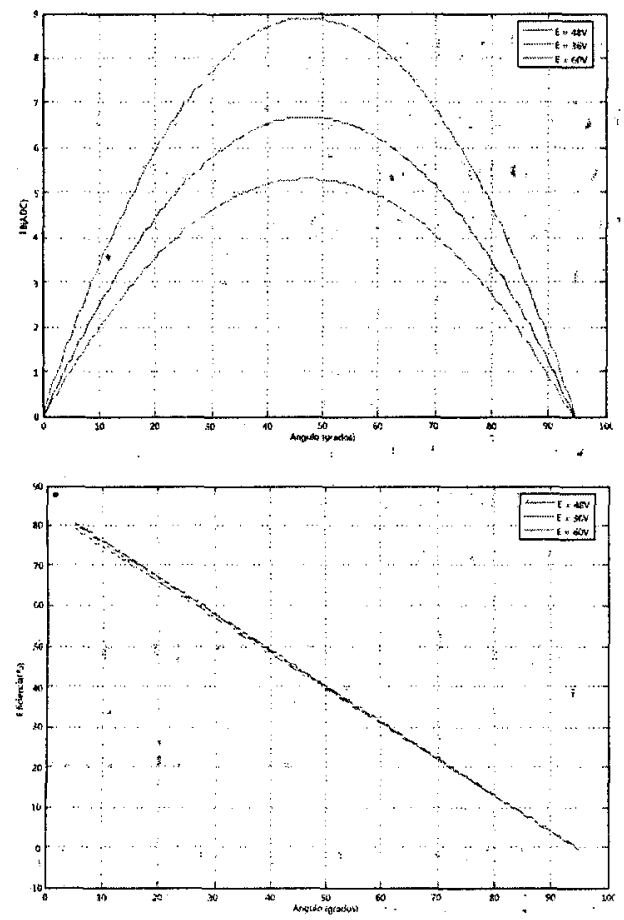

Figura 10 Con $V_{t h}=120 V_{r m s}$ a) Corriente en la batería $l_{B}$ vs. ángulo de desfasamiento para $E=36 \mathrm{~V}_{\mathrm{DC}}, 48 \mathrm{~V}_{\mathrm{DC}}$ y $60 \mathrm{~V}_{\mathrm{DC}}$. b) Eficiencia vs. ángulo de desfasamiento para $E=36$ VDC, 48 VDC y 60 VDC. 
De la figura 10 se observa que en las corrientes máximas para las cuales corresponde una potencia máxima de $324 \mathrm{~W}$, aproximadamente, el equipo presenta una eficiencia del $44 \%$.

De lo anterior se deduce que se puede transferir más potencia desde la red a las baterías variando el ángulo de desfasamieno $\varphi$.

\section{Modos de operación}

La unidad de conversión tiene básicamente tres modos de operación.

Modo voltaje AC constante

En este modo, la unidad de conversión mantiene el voltaje en $120 \mathrm{~V}_{\mathrm{rms}}$, siempre y cuando la potencia exigida no sea mayor de $400 \mathrm{~W}$. En caso que el equipo esté sometido a sobrecarga, pasa automáticamente al modo corriente AC constante hasta cuando desaparezca la sobrecarga.

\section{Modo corriente $\mathrm{AC}$ constante}

Se presenta automáticamente en la unidad en caso de sobrecarga; es decir, esta función actúa como un limitador de corriente. Cuando la unidad de conversión se encuentra en modo corriente $\mathrm{AC}$ constante suministra una corriente cercana a los $4 \mathrm{~A}_{\mathrm{rms}}$.

\section{Modo red}

Es el modo de operación de interconexión con la red pública. Está en capacidad de entregar y de recibir energía. También se puede colocar en estado de flotación, con flujo de energía nulo.

La transferencia de energía se logra variando de manera controlada parámetros propios de la unidad, como amplitud y fase, lo que lleva a que se presenten tres estados de transferencia de energía, los cuales se citan a continuación:

- Estado de flotación. Cuando el índice de modulación y el ángulo de desfasamient $\delta \varphi$ son tales que no se presenta intercambio de energía. Para este caso, el voltaje de salida de la unidad es igual al voltaje de la red en magnitud y fase. 
- Estado de generación. En este modo, la energía fluye desde las baterías hacia la red. El ángulo de desfasamiento $\varphi$ de la unidad se adelanta con respecto al de la red entregando potëncia activà.

- Estado de carga. En este modo de óperación, la energia fluye desde la red hacia las baterías. El ángulo de desfasamiento $\varphi$ de la unidad se atrasa con respecto al de la red recibiendo potencia activa para la carga de las baterías.

\section{Operación de unidades en paralelo}

En sistemas aislados que requieran potencias mayores a la capacidad nominal de la unidad de conversión, se pueden interconectar varias unidades en paralelo. La sincronización del sistema se puede realizar con un equipo

- Maestro y uno o varios esclavos con capacidad de generar salidas en fase con - la salida del equipo Maestro.

\section{CARACTERÍSTICAS DE DESEMPEÑO DE LA UNIDAD \\ Característica en vacío del conversor para diferentes valores de alimentación DC}

Esta prueba se llevó a cabo aplicando diferentes índices de modulación para tres valores de batería $\left(E=36.4 \mathrm{~V}_{\mathrm{DC}}, 47 \mathrm{~V}_{\mathrm{DC}}\right.$ y $\left.61 \mathrm{~V}_{\mathrm{DC}}\right)$. Se midieron los voltajes de salida sin carga, obteniendo los resultados que se muestran en la figura 11.

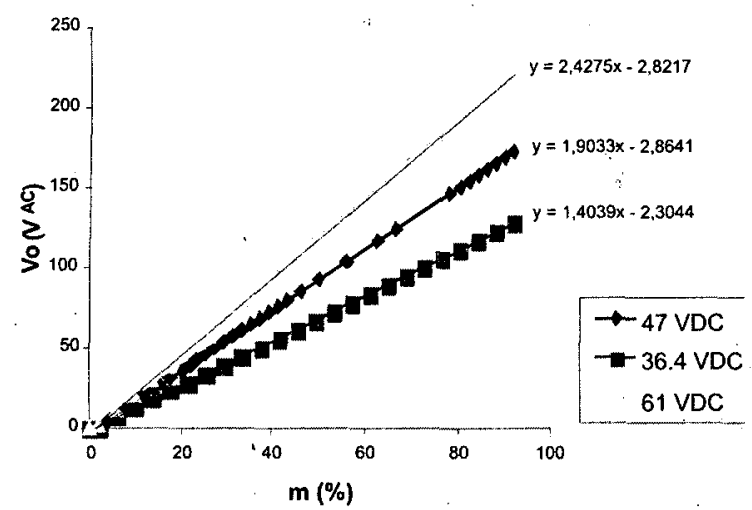

- Figura 11 Característica en vacío de la unidad. 
A partir de la gráfica se concluye que el efecto de variar $E$, se traduce en un cambio de pendiente en la característica de vacío de la unidad, que era de esperarse, porque el voltaje de salida es directamente proporcional a $E$.

\section{Características en carga e impedancia equivalente de la unidad}

Para determinar la magnitud de las impedancias del sistema, se procedió a elaborar una serie de mediciones de voltaje versus. corriente de carga para diferentes índices de modulación.

Los puntos sobre los cuales se tomaron medidas de voltaje fueron la salida del puente, la salida del filtro y la salida del transformador. Estos voltajes se midieron para diferentes corrientes de salida hasta llegar a la corriente nominal. A continuación se muestra la gráfica para $m=68.4 \%$.

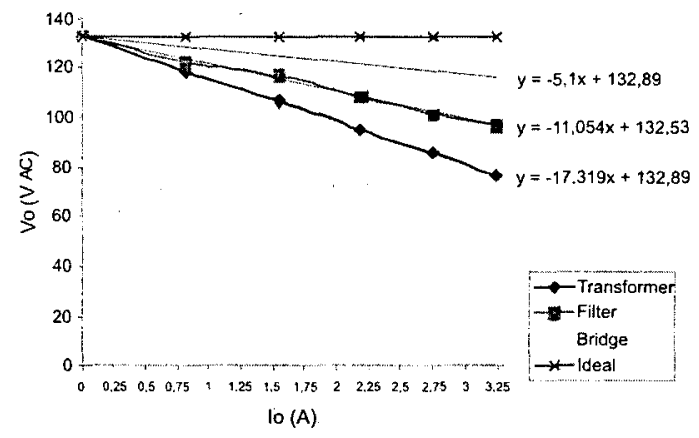

Figura 12 Característica de carga para $\mathrm{m}=68.4 \%$.

En la figura 12, la magnitud de la impedancia del equipo es aproximadamente $17.6 \Omega$ referidos al secundario del transformador.

\section{Eficiencia de la unidad}

Con carga resistiva pura de $400 \mathrm{~W}$ y corriente de $3.33 \mathrm{~A}_{\text {rms }}$, la potencia de entrada $\mathrm{P}_{i}$ es

$$
P_{i}=P_{o}+I_{o}^{2} * R=400+\left(3.333^{2} * 17.6\right)=595.16 \mathrm{~W}
$$

Finalmente, la eficiencia es

$$
\eta=\frac{P_{o}}{P_{o}+P_{\text {Pérdidas }}} * 100=67.2 \%
$$




\section{Parámetros de la unidad de conversión}

A continuación se presentan los principales parámetros de la unidad de conversión, así como la captura en el osciloscopio de la forma de onda de la tensión de salida, atenuada por 10.

Tabla 3.

Parámetros de la unidad de conversión

\begin{tabular}{|l|c|c|}
\hline \multicolumn{1}{|c|}{ Parámetros } & Valor & Unidad \\
\hline Impédancia & 17,6 & Ohms \\
\hline Eficiencia & 68 & $\%$ \\
\hline $\begin{array}{l}\text { Potencia Máx. de } \\
\text { Salida }\end{array}$ & 400 & $W$ \\
\hline THD & 1,3 & $\ldots$ \\
\hline HF3 & 1,15 & $\%$ \\
\hline HF5 & 0,5 & $\%$ \\
\hline HF7 & 0,326 & $\%$ \\
\hline
\end{tabular}

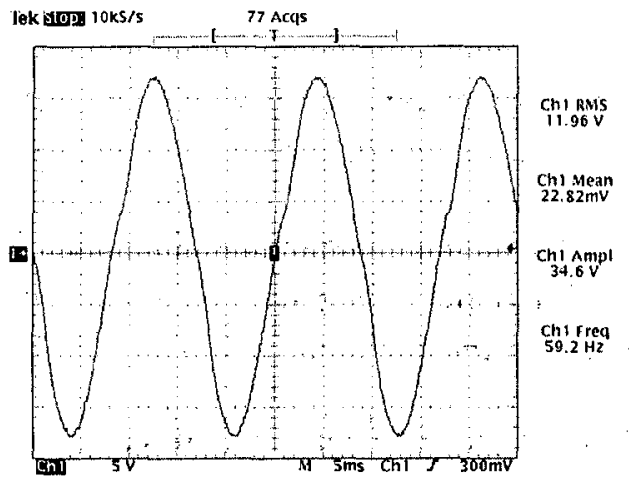

Figura 13 Forma de onda de tensión de salida.

Como se observa en la figura 13, la tensión de salida es una señal de 120VAC a $60 \mathrm{~Hz}$, con baja distorsión armónica.

\section{CONCLUSIONES}

Los conversores DC/AC con SPWM facilitan la interconexión de sistemas de generación a partir de fuentes de energía no convencionales a redes alimentadas con sistemas de generación tradicionales. 
En redes aisladas, el servicio se puede mejorar, en horas de baja generación, con sistemas de almacenamiento de energía que en algunos casos pueden ser bancos de baterías.

La tecnología SPWM presenta la gran ventaja de generar ondas sinusoidales con un bajo índice de distorsión armónica, y puede implementarse de manera rápida y sencilla. El prototipo construido puede conectarse directamente cuando la red está energizada, debido a que posee circuitos de control que permiten sincronizarse de manera rápida y segura.

En desarrollos futuros, es conveniente trabajar con conversores trifásicos y aplicar otros tipos de tecnologías de modulación a fin de lograr mayores ventajas en aplicaciones relacionadas con redes de generación distribuida y redes de fuentes de energía no convencional.

\section{BIBLIOGRAFÍA}

[1] V. Gabriunas, Apuntes de electrónica", Bogotá, Universidad Distrital Francisco José de Caldas 2000, p. 355.

[2] F. Marder, Generación de onda sinoidal. Modulación por ancho de pulso corriente, Department of Electric Engineering, Faculty of Exact and Natural Sciences and Surveying, UNNE. Corrientes, 1999, p. 4.

[3] M. Rashid, Electrónica de potencia, 3rd ed., México, McGraw-Hill, 1995, p. 702.

[4] J. Gualda, S. Martínez y P. Martínez, Electrónica industrial: técnicas de potencia. 2nd ed., Madrid, Alfaomega Marcombo, 1992, p. 477.

[5] Y. Zhang, "Snubber Considerations for IGBT Applications Power". International Rectifier. Application Note, International Versión. p. 7.

[6] J. Grainger y W. Stevenson, Análisis de sistemas de potencia, México, McGraw-Hill, 1996, p. 740. 